\title{
Predicting the sound insulation of finite double-leaf walls with a flexible frame
}

\author{
Jan C.E. Van den Wyngaert ${ }^{\mathrm{a}}$, Mattias Schevenels ${ }^{\mathrm{b}}$, Edwin P.B. Reynders ${ }^{\mathrm{a}}$
}

${ }^{a}$ KU Leuven Faculty of engineering science: Department of Civil Engineering, Kasteelpark Arenberg 40, B-3001 Leuven, Belgium.

${ }^{b}$ KU Leuven Faculty of engineering science: Department of Architecture, Kasteelpark Arenberg 1, B-3001 Leuven, Belgium

\begin{abstract}
With double-leaf wall systems such as plasterboard walls, a high sound insulation can potentially be achieved with a relatively low weight. The accurate sound transmission analysis of this type of wall is challenging since the leafs are usually coupled to a common frame, and since the finite dimensions play a role at lower frequencies. Existing analytical models for sound insulation prediction account for the deformation of the wall in an approximate way, while detailed numerical models are computationally very demanding. In this work, a sound insulation prediction model that achieves a high prediction accuracy at a low computational cost is developed. The wall components that display low geometrical complexity, such as the wall leafs and the cavity, are modelled in a analytical way. Sound absorbents in the cavity are modelled as equivalent fluids. The metal studs, which have a highly deformable cross section, are modelled in full detail with finite elements. The sound fields in the sending and receiving rooms are modelled as diffuse; they are rigorously coupled to the deterministic wall model by employing a hybrid deterministic-statistical energy analysis framework. With the resulting room-wall-room model, the airborne sound insulation is predicted for a range of double-leaf plasterboard walls with single, double and triple plating and with different cavity depths. The obtained transmission losses are validated against the results of an extensive set of experimental tests. A very good agreement between predicted and measured transmission loss values is observed. The single number ratings for the airborne sound insulation for nearly all walls differ from the experimental values by 0 to $2 \mathrm{~dB}$, which is close to the average experimental reproducibility. At the same time, the computational cost is more than three orders of magnitude lower than for recently proposed models of similar accuracy.
\end{abstract}

Keywords:

airborne sound insulation, hybrid deterministic-statistical energy analysis, double wall systems, plasterboard walls, flexible metal stud frame

\section{Introduction}

Double-leaf wall systems are often used in the construction, aerospace, railway, and other industries because of their light weight and potentially high sound insulation. Even when the walls are not load bearing, the leafs are usually coupled to a common frame for reasons of cost and lateral stability. A typical example is a double-leaf plasterboard wall where the leafs are screwed into a common metal stud frame. Walls of this type are very often employed in the construction industry for partitioning large open spaces into smaller rooms. However, the common frame introduces a structural transmission path between both leafs in addition to the airborne path through the cavity. The sound insulation of this type of wall is therefore lower than for a wall with structurally decoupled leafs, at least above the mass-spring-mass resonance frequency of the decoupled system, where the leafs vibrate as rigid masses, compressing the cavity fluid. The prediction of the airborne sound insulation of double studded walls is challenging since it is characterized by many parameters and physical phenomena. Experimental parametric studies by Hongisto et al. [1], Vermeir and Gerretsen [2], and Mechel and Royar [3] have shown that the airborne sound insulation of a decoupled double wall depends mainly on the individual plate thicknesses, the number of plates, the combination of different thicknesses and plate materials, the cavity depth, and the wall dimensions. The stud geometry, the number of studs and the number of screws per stud are additional parameters influencing the sound insulation when the leafs are coupled. The mass-spring-mass resonance is both defined by the total weight of the leafs and the cavity depth. The coincidence frequency, at which the wavelength of the incident wave and the bending wavelength of a leaf are equal, depends on 
the material parameters of the leaf and its thickness. All these different phenomena and input parameters make it hard to accurately predict the airborne sound insulation using a simplified model.

Many models for the prediction of the airborne sound insulation of double-leaf walls exist. A concise review of these models is given in Chapter 2. They can be subdivided into analytical models and numerical models. Analytical models are often used to gain insight into the main physical phenomena, e.g. the mass-spring-mass resonance and coincidence. A low computational cost is achieved by assuming diffuse conditions in the transmission rooms and by using simplified models for the vibrational behavior of the leafs and the cavity. The prediction of the airborne sound insulation is often inaccurate due to this simplified representation of the wall components and since some physical phenomena are omitted, e.g. the dynamic behavior of the studs. An overview of these models is given in Section 2.1 .

Numerical models, e.g finite element models, manage to capture the vibratory behavior of both the wall and the transmission rooms in full detail, making it possible to accurately predict the single number rating. However, these models are not practical due to the large number of boundary and/or finite elements needed at high frequencies. An overview of these models is given in Section 2.2.

The goal of this work is to close the gap between these two types of models. A prediction model for the airborne sound insulation of double walls is developed with both a high prediction accuracy and a low computational cost. Due to this low computational cost and high prediction accuracy, the model can be used as a design tool for the sound insulation of double walls.

The wall is modelled deterministically to accurately capture its vibratory behavior while the sound fields in the transmission rooms are modelled as diffuse. The wall components that display low geometrical complexity, such as the wall leafs and the cavity, are modelled in an analytical way to reduce the computational cost. When a single leaf consists of multiple plates, an expression of the equivalent bending stiffness of the composite leaf is used. For a wall with soft sound absorbents in the cavity, an equivalent fluid model for the acoustic wave propagation inside the cavity is employed. The metal studs, which have a highly deformable cross section, are modelled in full detail with finite elements. The hybrid Deterministic-Statistical Energy Analysis (DET-SEA) framework [4, 5] is adopted to rigorously couple the deterministic wall model to the diffuse room models by employing the diffuse field reciprocity relationship [6]. The prediction model presented in this work is validated against the results of an extensive set of experimental tests of double plasterboard walls with a common flexible stud frame [2].

The remainder of this article is structured as follows. A review of existing prediction models is given in section 2. They are divided in analytical models and numerical models. The deterministic model of the double wall and the coupling to the sound fields in the adjacent transmission rooms is elaborated in section 3 . In section 4 , the experimental setup and test results for the measurements of the sound insulation of studded double plasterboard walls are presented. The results of the model are extensively compared with experimental results for double plasterboard walls with a single stud frame. Conclusions and final remarks are listed in section 5.

\section{Review of existing models}

A concise review of existing models for the prediction of the airborne sound insulation of double-leaf walls is provided here. They are subdivided into two categories. The analytical models are based on simplified representations of the leafs, the cavity and the transmission rooms. The numerical models mainly use finite elements and/or boundary elements to predict the sound insulation.

\subsection{Analytical models}

Early analytical models for the airborne sound insulation of double-leaf walls concentrated on the airborne transmission path through walls with very large planar dimensions. Structural connections between the wall leafs were not considered, and the leafs themselves were modelled as being of infinite extent. The first models such as the one of Beranek and Work [7] considered only normally incident sound waves and therefore included only the mass of the leafs and the width of the cavity. They allowed for a qualitative study of physical effects such as the mass-spring-mass resonance of the leafs onto the compressive air cavity, and standing waves in the cavity. In 1950, London introduced a model for the sound transmission under obliquely incident sound waves, in which also the stiffness and dissipation of the leafs plays a role [8]. This model therefore includes additional physical effects such as coincidence. The diffuse transmission loss in the model of London is obtained from the Paris equation, i.e., as a weighted summation of the plane-wave oblique transmission loss over all angles of incidence. 
Mulholland and coworkers extended the model of Beranek and Work to include oblique incidence [9] and later on extended their model by including multiple reflections of sound in the cavity $[10,11]$. This so-called multiple reflection model can be used to examine the effects of absorption in the cavity. The absorption is taken into account using a damping ratio on the reflected waves either on the plate-cavity interface or on the cavity edges. Heckl went back to the original model of Beranek and Work to include sound absorbing materials based on the stiffness per unit area, $s^{\prime}$, of the material inside the cavity [12]. He modelled the sound insulation of double walls consisting of two thin plates with a resilient material in between. This model showed good results for highly absorbing cavities but underestimated the sound insulation for empty cavities. Fahy $[13,14]$ introduced cavity absorbents in the model of Beranek and Work for pure normal incidence using an equivalent fluid model for the wavenumber and the density of the air in the cavity. Au and Byrne $[15,16]$ introduced expressions for the impedances of porous layers, impervious barriers, and air spaces. Kang et al. [17] compared results of the previous models with experimental data and found that the angles close to grazing incidence were attenuated better than angles close to normal incidence. By introducing a Gaussian weighting on the Paris equation, they were able to predict the sound insulation better for empty cavities. For absorbing cavities the angles close to grazing incidence were already more attenuated so the Gaussian weighting was not needed. At low frequencies, the previously discussed models deviate from the experimental results due to the modal behavior of the wall. Donato [18] suggested a correction for the finite size of the wall using a wavenumber approach for the models of Beranek and Work, and London. This correction can be applied to all previous models that assume infinite panel size. He also simplified the integrals obtained in the Paris equation both in the works of Beranek and Work, and London so that they could be evaluated analytically.

In the previous models, structural connections through the studs are not taken into account. Rigid connections between the wall leafs, so-called 'sound bridges', were included by Sharp [19, 20] and later by Cremer and Heckl [21]. These models divide the transmission of the sound through the wall in a structure-borne and an airborne path with the assumption that both paths do not influence each other. Sharp adopted the model of London for the calculation of the transmission through the airborne path. Above the so-called bridge frequency, i.e. the frequency where the transmission trough the stud and through the cavity are equal, a correction for the transmission through the rigid stud is introduced. Lin and Garrelick [22] derived an analytical solution for the sound insulation of double walls of infinite extent with periodically spaced rigid studs. The connections between the plates and the rigid studs are modelled as springs. The interaction between the studs and the pressure field inside the cavity was not included. The approach was later adapted by Brunskog [23]. In his model, the interactions between the rigid studs and the pressure field in the cavity were taken into account. Several authors, including Gu and Wang [24], Wang et al. [25], Poblet-Puig et al. [26], Davy [27, 28, 29], Vigran [30] and Guigou-Carter et al. [31], have extended analytical models to include flexible studs. The studs are then modelled either as a series of decoupled point springs [31], as line springs [24, 26, 29, 30], or as a smeared elastic layer [25]. Some authors include only a translational spring stiffness [24, 29, 30, 31], others also include a rotational stiffness $[25,26]$. This type of flexible stud modelling is very approximate; therefore empirical, frequency-dependent values for the spring stiffness are employed [26, 29].

In the previous analytical models, the wall is modelled deterministically and the sound fields in the transmission rooms are assumed to be diffuse. In Statistical Energy Analysis (SEA), the wall is decomposed into homogeneous subsystems, e.g. the plates, with the assumption that each subsystem carries a diffuse field. Each subsystem is characterized by only a single parameter, its total energy. Steady-state energy flows between the subsystems are modelled approximately. The airborne sound insulation can be calculated from the ratio of the total energy of the transmission and the receiving room. White and Powell [32] modelled the sound insulation of a double-leaf wall based on the SEA approach of Lyon and Maidanik [33], in which the coupling loss factors between the subsystems are based on the work of Lyon and Scharton [34]. Crocker and Price included the mass law in the model of White and Powell by introducing a non-resonant transmission path of the mass-impeded modes of the plate [35]. This gives rise to a coupling loss factor directly between the transmission rooms and the cavity. The absorption in the cavity is taken into account as in the model of Cummings and Mulholland [9], by applying absorption either at the plate-cavity boundary or at the cavity edges. Craick and Smith extended the SEA model of Crocker and Price taking into account a rigid wooden stud frame $[36,37]$. The coupling loss factors for both line and point connections were based on the work of Cremer and Heckl [21]. The main disadvantage of the models is that the stiffness of the air inside the cavity is ignored. Therefore, the mass-air-mass resonance is not present in these models. 


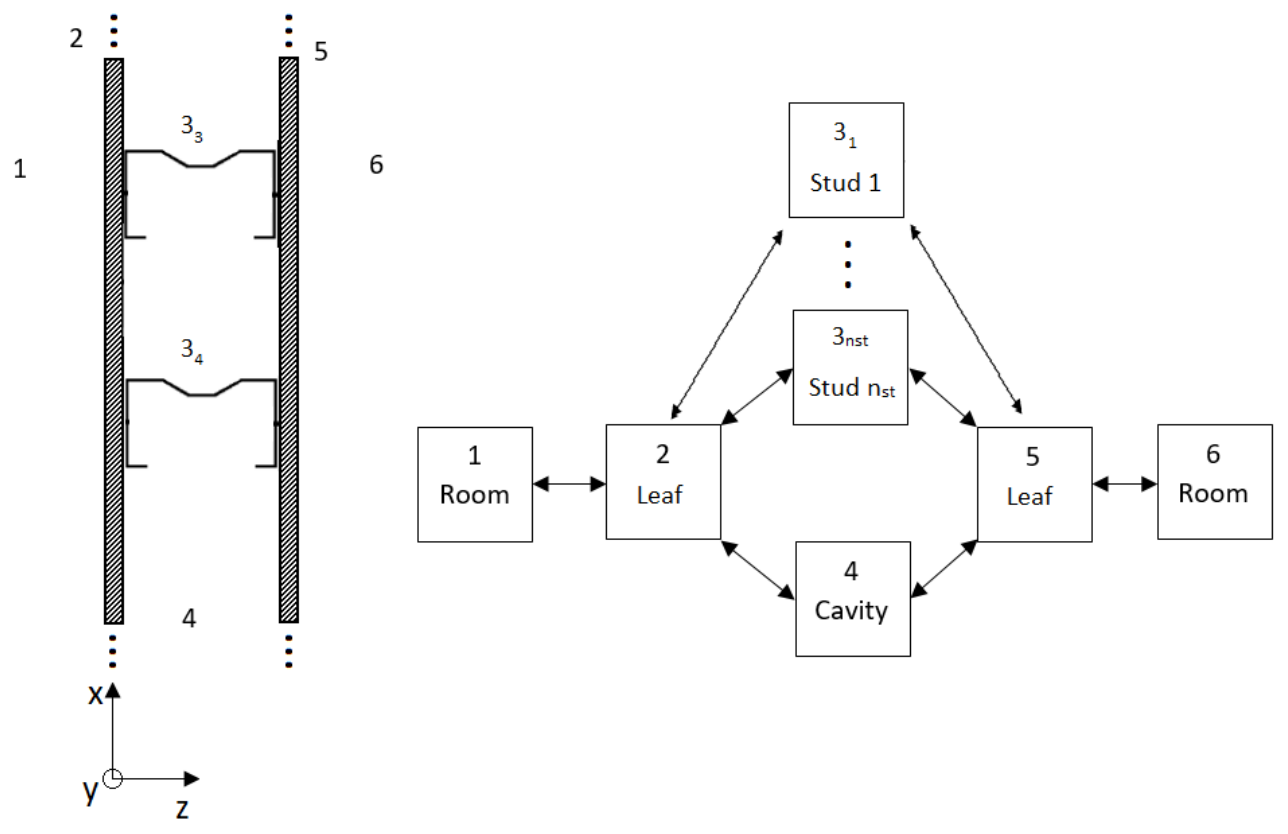

Figure 1: Components of a double wall with a flexible stud frame.

\subsection{Numerical models}

The analytical models often omit certain physical phenomena and lead to either a relatively poor prediction accuracy or they are only applicable for certain wall types, e.g. walls with empty cavities or without studs. Element-based numerical methods can achieve a better prediction accuracy at the expense of high computational costs. The modelling of the airborne sound insulation by finite elements became possible in the 1990s due to the increase of computational power. Since these models were still computationally costly, simplifications were made. By assuming semi-infinite spaces for the transmission rooms and omitting the studs, the computational cost was reduced. These simplified models only hold at low frequencies since the low frequency sound insulation is mostly dominated by the sound transmission though the cavity path. The disadvantage of the models is that a fine mesh and a large number of eigenmodes are needed for the computation of the sound insulation at higher frequencies.

Atalla and Panneton, and Atalla and Sgard modelled the low frequency sound insulation based on a finite element formulation of the different layers of the wall coupled with a boundary element method to account for fluid loading $[38,39]$. Legault and Atalla [40] extended these models to include the coupling of the leafs with a frame using the mount stiffness, accounting for perfectly rigid, resilient and flexible mounts. In this model both the displacements and the rotations of the frame and the leafs were coupled using structural constraint equations. Davidsson and Brunskog included sheet metal studs in their finite element model for a better approximation at higher frequencies where the structure borne path can not be omitted [41, 42]. This model was only used in frequency ranges up to a maximum of $630 \mathrm{~Hz}$ due to the large computational demand. Arjunan et al. extended the frequency range up to $3150 \mathrm{~Hz}$ [43, 44]. To reduce the computational cost, the sound insulation is only evaluated at three frequencies ranging from $\pm 5 \%$ of the center frequencies of the $1 / 3$ octave bands. The sound insulation is averaged over these three frequencies to include the oscillatory behavior.

\section{An efficient and accurate model}

From the previous discussion, it is clear that existing models for the sound insulation prediction of double-leaf walls are either insufficiently accurate (analytical models) or they have a very high computational cost (numerical models). Therefore, in this section, a forward model with both a high prediction accuracy and a low computational cost is developed. 
The wall is modelled deterministically to capture its vibration behavior in full detail while the sound fields in the sending and receiving rooms are modelled as diffuse. The components of the model are represented in Fig. 1. The wall components that display low geometrical complexity, i.e. the wall leafs and the cavity, represented as components 2, 4 and 5 in Fig. 1, are modelled in an analytical way to reduce the computational cost. The metal studs, shown as part $3_{1}$ till $3_{\mathrm{n}_{\mathrm{st}}}$ in Fig. 1, are modelled in full detail with finite elements. The hybrid DET-SEA modelling framework $[4,5]$ is used to rigorously couple the diffuse sound fields in the transmission rooms, represented as components 1 and 6 in Fig. 1, to the deterministic wall model by employing the diffuse field reciprocity relationship [45]. In what follows, the deterministic model of the wall system is explained first. Subsequently, the interaction between the wall and the sound fields in the adjoining rooms is described.

\subsection{Deterministic model of the wall}

The considered double wall system consists of two leafs separated by an air cavity and coupled with a common stud frame. In the first instance, the leafs, the cavity and the individual studs are decoupled from each other. The assumedmodes method [46] is then used for approximating the vibration field of the two wall leafs, $u_{11}$ and $u_{12}$, the pressure field within cavity, $\mathrm{p}_{\mathrm{cav}}$, and the vibration field of the stud number $l, \mathrm{u}_{\mathrm{st}, l}$, at spatial location $\mathrm{x}$ and frequency $\omega$, using a finite set of basis functions $\phi$, that satisfy the boundary conditions, and corresponding generalized coordinates $q$ :

$$
\begin{aligned}
& u_{11}(\mathbf{x}, \omega) \approx \sum_{k=1}^{n_{1}} \phi_{11, k}(\mathbf{x}) q_{11, k}(\omega)=\phi_{11}(\mathbf{x}) \mathbf{q}_{11}(\omega) \\
& u_{12}(\mathbf{x}, \omega) \approx \sum_{k=1}^{n_{2}} \phi_{12, k}(\mathbf{x}) q_{12, k}(\omega)=\phi_{12}(\mathbf{x}) \mathbf{q}_{12}(\omega) \\
& p_{\mathrm{cav}}(\mathbf{x}, \omega) \approx \sum_{k=1}^{n_{\mathrm{cav}}} \phi_{\mathrm{cav}, k}(\mathbf{x}) q_{\mathrm{cav}, k}(\omega)=\phi_{\mathrm{cav}}(\mathbf{x}) \mathbf{q}_{\mathrm{cav}}(\omega) \\
& u_{\mathrm{st}, l}(\mathbf{x}, \omega) \approx \sum_{k=1}^{n_{\mathrm{st}, l}} \phi_{\mathrm{st}, l, k}(\mathbf{x}) q_{\mathrm{st}, l, k}(\omega)=\phi_{\mathrm{st}, l}(\mathbf{x}) \mathbf{q}_{\mathrm{st}, l}(\omega) .
\end{aligned}
$$

The choice of the basis functions $\phi$ of the decoupled components will be discussed in detail below. The vibration fields, $\mathrm{u}_{11}, \mathrm{u}_{12}$ and $\mathrm{u}_{\mathrm{st}, l}$, denote the out-of-plane displacements of the wall components. Subsequently, the interaction between the components will be achieved by introducing coupling loads between the cavity and the leafs and by constraining the leaf and stud displacements to be equal at the connection locations. This will result in a system of equations in which the generalized coordinates of the wall components are coupled:

$$
\left[\begin{array}{ccccccc}
\mathbf{D}_{11} & \mathbf{0} & \mathbf{L}_{\mathrm{f} 1} & \mathbf{0} & \ldots & \mathbf{0} & \mathbf{C}_{11}^{T} \\
\mathbf{0} & \mathbf{D}_{12} & \mathbf{L}_{\mathrm{f} 2} & \mathbf{0} & \ldots & \mathbf{0} & \mathbf{C}_{12}^{T} \\
\mathbf{L}_{\mathrm{s} 1} & \mathbf{L}_{\mathrm{s} 2} & \mathbf{D}_{\mathrm{cav}} & \mathbf{0} & \cdots & \mathbf{0} & \mathbf{0} \\
\mathbf{0} & \mathbf{0} & \mathbf{0} & \mathbf{D}_{\mathrm{st}, 1} & \cdots & \mathbf{0} & \mathbf{C}_{1}^{T} \\
\vdots & \vdots & \vdots & \vdots & \ddots & \vdots & \vdots \\
\mathbf{0} & \mathbf{0} & \mathbf{0} & \mathbf{0} & \ldots & \mathbf{D}_{\mathrm{st}, n_{\mathrm{st}}} & \mathbf{C}_{\mathrm{n}_{\mathrm{st}}^{T}}^{T} \\
\mathbf{C}_{11} & \mathbf{C}_{12} & \mathbf{0} & \mathbf{C}_{1} & \cdots & \mathbf{C}_{\mathrm{n}_{\mathrm{st}}} & \mathbf{0}
\end{array}\right]\left[\begin{array}{c}
\mathbf{q}_{11} \\
\mathbf{q}_{12} \\
\mathbf{q}_{\mathrm{cav}} \\
\mathbf{q}_{\mathrm{st}, 1} \\
\vdots \\
\mathbf{q}_{\mathrm{st}, n_{\mathrm{st}}} \\
\boldsymbol{\lambda}
\end{array}\right]=\left[\begin{array}{c}
\mathbf{f}_{11} \\
\mathbf{f}_{12} \\
\mathbf{0} \\
\mathbf{0} \\
\vdots \\
\mathbf{0} \\
\mathbf{0}
\end{array}\right]
$$

or shortly,

$$
\mathbf{D}_{\mathrm{d}} \mathbf{q}=\mathbf{f} .
$$

In Eq. (5), $\mathbf{D}_{11}, \mathbf{D}_{12}, \mathbf{D}_{\text {cav }}$ and $\mathbf{D}_{\mathrm{st}, l}$ represent the dynamic stiffness matrices of the first wall leaf, the second wall leaf, the cavity and stud number $l$, respectively; $\mathbf{L}_{\mathrm{f} 1}$ and $\mathbf{L}_{\mathrm{f} 2}$ account for the fluid loading (acoustic pressure) of the cavity onto the first and the second wall leaf, respectively; $\mathbf{L}_{\mathrm{s} 1}$ and $\mathbf{L}_{\mathrm{s} 2}$ account for the structural vibration loading by the first and second wall leaf, respectively, onto the cavity fluid; the $\mathbf{C}$ matrices are matrices that are employed for constraining the stud and wall displacements at their connection locations, and $\boldsymbol{\lambda}$ collects the corresponding Lagrange multipliers; and finally the f matrices represent external fluid loading, by the acoustic pressure in the adjoining rooms, onto the wall leafs. All matrices introduced above will be discussed in detail in the following sections. 


\subsubsection{Analytical model of the wall leafs}

Since the leafs of the considered wall system are thin, the Kirchoff-Love thin plate theory is adequate for modelling their dynamic behavior. Furthermore, the assumption of simply supported boundary conditions along the edges of the plate is fairly accurate in practice [39, 40, 41]. It follows from these considerations that the vibration behavior of the wall leafs, when decoupled from all other wall components, can be modelled analytically. This is illustrated in the present section for the first wall leaf; the same lines can be followed for the second leaf.

The basis functions which appear in (1) are taken to be the mass-normalized mode shapes of the decoupled, thin, rectangular, simply supported wall leaf:

$$
\phi_{11, k}(\mathbf{x})=\frac{2}{\sqrt{m_{11}}} \sin \left(\frac{q_{k} \pi x}{L_{\mathrm{x}}}\right) \sin \left(\frac{r_{k} \pi y}{L_{\mathrm{y}}}\right),
$$

where $m_{11}$ denotes the total mass of the leaf, $L_{\mathrm{x}}$ and $L_{\mathrm{y}}$ denote its planar dimensions in the $x$ and $y$ coordinate directions, respectively, and $q_{k} \in \mathbb{N}_{0}$ and $r_{k} \in \mathbb{N}_{0}$ denote the number of half wavelengths in those directions, specific for mode number $k$. The modes are numbered according to increasing natural frequency. The angular natural frequency of mode $k$ equals

$$
\omega_{11, k}=\sqrt{\frac{D_{11}}{m_{11}^{\prime \prime}}}\left(\left(\frac{q_{k} \pi}{L_{\mathrm{x}}}\right)^{2}+\left(\frac{r_{k} \pi}{L_{\mathrm{y}}}\right)^{2}\right),
$$

where $D_{11}$ denotes the bending stiffness of the leaf, and $m_{11}^{\prime \prime}$ its mass per unit surface area.

The leaf may consist of a single thin plate, or of multiple plates on top of each other. In the latter case, it will be assumed that there are no shear connections between the plates, such that perfect slip conditions exist at the plate-plate interfaces. In this way the bending stiffness of the leaf is slightly underestimated since in reality the plates are jointly screwed into the flexible studs at discrete points. However, Davidsson and Brunskog [42] have shown that the perfect slip assumption is fairly accurate, so the bending stiffness of the entire leaf can be obtained from

$$
D_{11}=\sum_{s=1}^{n_{\mathrm{p} 11}} D_{\mathrm{pl}, s}
$$

where $n_{\mathrm{pl1}}$ denotes the total number of plates from which the considered leaf is composed, and $D_{\mathrm{pl}, s}$ denotes the dynamic bending stiffness of plate number $s$ :

$$
D_{\mathrm{pl}, s}=\frac{E_{s} \mathrm{t}_{\mathrm{s}}{ }^{3}}{12\left(1-\nu_{s}^{2}\right)}
$$

In this equation, $E_{s}$ denotes the Young's modulus of plate $s, \nu_{s}$ its Poisson's ratio, and $t_{s}$ its thickness.

Since the basis functions have been chosen to be the mass-normalized mode shapes of the leaf, the corresponding generalized coordinates are the modal amplitudes of the leaf. Its equation of motion therefore reads

$$
\mathbf{D}_{11} \mathbf{q}_{11}=\tilde{\mathbf{f}}_{11},
$$

where $\mathbf{D}_{11}$ is a diagonal matrix with entries

$$
D_{11, k k}=-\omega^{2}+\omega_{11, k}^{2}\left(1+\mathrm{i} \eta_{11, k}\right),
$$

where $\mathrm{i}:=\sqrt{-1}$ is the imaginary unit and $\eta_{11, k}$ denotes the damping loss factor of mode $k$. When all loading onto the wall leaf as well as any constraints are incorporated within the modal load vector $\tilde{\mathbf{f}}_{11}$, (11) is fully consistent with the first block row of the equation of motion of the complete wall system, Eq. (5).

\subsubsection{Analytical model of the cavity}

For the sound field within the cavity, the conventional linear acoustic theory is adopted. Since there are no sound sources within the cavity itself, the acoustic pressure within the cavity $\mathrm{p}_{\text {cav }}(\mathbf{x}, \omega)$ satisfies the homogeneous 
Helmholtz equation. The interaction between the sound field in the cavity and the studs is neglected, i.e., the studs are assumed to be acoustically transparent, such that the cavity has a rectangular cuboid shape. This is a common assumption in sound transmission models of double-leaf walls, see e.g. Lin and Garrelick [22], and Davy [27, 28, 29]. Since the particle velocity at the boundaries is small, hard-walled boundary conditions are assumed. This assumption introduces a discontinuity between the particle velocity inside the cavity and the velocity of the wall leafs at the cavity-leaf boundary interfaces, yet with negligible error on the cavity pressure field [13]. The interaction between the wall leafs and the cavity is therefore not modeled by imposing continuity of the velocity fields, but by taking the displacement fields of the wall leafs as external loads onto the cavity pressure field, and vice versa. These interactions will be detailed further on.

From these considerations, it follows that the sound pressure field within the cavity can be modelled analytically. The basis functions which appear in (3) are taken to be the normalized mode shapes of the decoupled, hard-walled, rectangular cuboid cavity [47]:

$$
\phi_{\mathrm{cav}, k}(\mathbf{x})=a_{k} \cos \left(\frac{m_{k} \pi x}{L_{\mathrm{x}}}\right) \cos \left(\frac{n_{k} \pi y}{L_{\mathrm{y}}}\right) \cos \left(\frac{p_{k} \pi z}{L_{\mathrm{z}}}\right),
$$

where $L_{\mathrm{z}}$ denotes the cavity depth, $m_{k} \in \mathbb{N}, n_{k} \in \mathbb{N}$ and $p_{k} \in \mathbb{N}$ denote the number of half wavelengths in the $x, y$ and $z$ coordinate directions. The normalization constant satisfies

$$
a_{k}=\frac{\mathrm{c} \gamma\left(m_{k}\right) \gamma\left(n_{k}\right) \gamma\left(p_{k}\right)}{\sqrt{L_{\mathrm{x}} L_{\mathrm{y}} L_{\mathrm{z}}}} \quad \text { where } \quad \gamma(s)= \begin{cases}\sqrt{2} & \text { if } s=0 \\ 1 & \text { if } s \neq 0\end{cases}
$$

and $c$ denotes the sound speed. The modes are numbered according to increasing natural frequency. The angular natural frequency of mode $k$ equals

$$
\omega_{\mathrm{cav}, k}=\mathrm{c} \sqrt{\left(\frac{m_{k} \pi}{L_{\mathrm{x}}}\right)^{2}+\left(\frac{n_{k} \pi}{L_{\mathrm{y}}}\right)^{2}+\left(\frac{p_{k} \pi}{L_{\mathrm{z}}}\right)^{2}} .
$$

Since the basis functions have been chosen to be the normalized mode shapes of the cavity, the corresponding generalized coordinates are the modal amplitudes of the cavity. Its equation of motion therefore reads

$$
\mathbf{D}_{\text {cav }} \mathbf{q}_{\mathrm{cav}}=\tilde{\mathbf{f}}_{\mathrm{cav}}
$$

where $\mathbf{D}_{\text {cav }}$ is a diagonal matrix with entries

$$
D_{\text {cav }, k k}=-\omega^{2}+\omega_{\text {cav }, k}^{2}\left(1+\mathrm{i} \eta_{\text {cav }, k}\right),
$$

and $\eta_{\text {cav }, k}$ denotes the damping loss factor of mode $k$. When the cavity is empty, the damping inside the cavity is prescribed in terms of a reverberation time $T_{\text {cav }}$. The modal loss factors can then be determined from [48, Ch 2.4.1]

$$
\eta_{\mathrm{cav}, k}=\frac{4.4 \pi}{\omega_{\mathrm{cav}, k} T_{\mathrm{cav}}}
$$

When the loading onto the cavity fluid is incorporated within the modal load vector $\tilde{\mathbf{f}}_{\mathrm{cav}},(16)$ is fully consistent with the third block row of the equation of motion of the complete wall system, Eq. (5).

Soft porous materials such as mineral wool are often placed inside the cavity to increase thermal and acoustic performance. When the deformation of the solid skeleton of the porous material is negligible, the cavity fluid model that was presented above can still be employed yet with modified, complex material properties. Several of these so-called equivalent fluid models have been proposed in the literature on an empirical basis. In the present work, the Delany-Bazley-Miki equivalent fluid model (DBM) is used; it is an extension of the Delany-Bazley-model [49] towards low frequencies. The DBM-model depends only on the flow resistivity $\sigma$ of the porous material and the 
frequency. When formulated in terms of a complex sound speed $c_{\mathrm{c}}$ and complex fluid density $\rho_{\mathrm{c}}$, this model reads

$$
\begin{aligned}
& c_{\mathrm{c}}=\frac{c}{1+7.81 a-\mathrm{i} 11.41 a}, \\
& \rho_{\mathrm{c}}=\frac{c \rho_{\mathrm{a}}}{c_{\mathrm{c}}}[1+5.50 b-\mathrm{i} 8.43 b], \\
& a=\left(10^{3} \frac{f^{\prime}}{\sigma^{\prime}}\right)^{-0.618} \text { and } b=\left(10^{3} \frac{f^{\prime}}{\sigma^{\prime}}\right)^{-0.632},
\end{aligned}
$$

where $f^{\prime}:=\frac{f}{1 \mathrm{~Hz}}$ denotes a dimensionless frequency, $\sigma^{\prime}:=\frac{\sigma}{1 \mathrm{Ns} / \mathrm{m}^{4}}$ a dimensionless flow resistivity and $\rho_{\mathrm{a}}$ the air density. Note that, when cavity damping is modelled with complex material properties, $\eta_{\mathrm{cav}, k}$ is taken to be zero in (17).

\subsubsection{Fluid-structure interaction at the cavity boundaries}

Let us now consider the part of the modal load onto the first wall leaf, $\tilde{\mathbf{f}}_{11}$, which is due to the cavity pressure $p_{\text {cav }}(\mathbf{x}, \omega)$. When a particular mode $k$ of the leaf is considered, this load equals

$$
f_{11, k, \operatorname{cav}}(\omega)=-\int_{\Gamma_{11}} \phi_{11, k}(\mathbf{x}) p_{\text {cav }}(\mathbf{x}, \omega) d \mathbf{x},
$$

where $\Gamma_{11}$ denotes the interface surface area between the leaf and the cavity. The minus sign results from the fact that for a positive cavity pressure, the force exerted onto the first wall leaf points in the negative $z$ direction (see Fig. 1). With the chosen set of basis functions for the cavity pressure, the above expression can be elaborated as

$$
f_{11, k, \mathrm{cav}}(\omega) \approx-\sum_{l=1}^{n_{\mathrm{cav}}} L_{\mathrm{f} 1, k l} q_{\mathrm{cav}, l}(\omega)
$$

where

$$
L_{\mathrm{f} 1, k l}:=\int_{\Gamma_{11}} \phi_{11, k}(\mathbf{x}) \phi_{\mathrm{cav}, l}(\mathbf{x}) d \mathbf{x} .
$$

Note that $L_{\mathrm{f} 1, k l}$ equals element $(k, l)$ of the coupling matrix $\mathbf{L}_{\mathrm{f} 1}$ from the overall system of equations (5). Substitution of (7) and (13) into (24) and evaluating the integral yields

$$
L_{\mathrm{f} 1, k l}=\frac{-2 a_{l} \mathrm{~L}_{\mathrm{x}} \mathrm{L}_{\mathrm{y}}}{\pi^{2} \sqrt{m_{11}}} \cos \left(\frac{p \pi z}{\mathrm{~L}_{\mathrm{z}}}\right) \mathrm{b}\left(q_{k}, m_{l}\right) \mathrm{b}\left(r_{k}, n_{l}\right),
$$

where

$$
\mathrm{b}(\alpha, \beta):= \begin{cases}\frac{\beta \cos (\alpha \pi) \cos (\beta \pi)-\beta+\alpha \sin (\alpha \pi) \sin (\beta \pi)}{\alpha^{2}-\beta^{2}} & \text { for } k \neq l \\ \frac{\sin (\alpha \pi)^{2}}{2 \alpha} & \text { for } k=l\end{cases}
$$

Subsequently, let us consider the part of the modal load onto the cavity, $\tilde{\mathbf{f}}_{\mathrm{cav}}$, which is due to displacement of the first wall leaf, $u_{11}(\mathbf{x}, \omega)$. When a particular mode $k$ of the cavity is considered, this load equals

$$
f_{\mathrm{cav}, k, 11}(\omega)=-\rho_{\mathrm{a}} \omega^{2} \int_{\Gamma_{11}} \phi_{\mathrm{cav}, k}(\mathbf{x}) u_{11}(\mathbf{x}, \omega) d \mathbf{x} .
$$

With the chosen set of basis functions for the leaf displacement, the above expression can be elaborated as

$$
f_{\mathrm{cav}, k, 11}(\omega) \approx-\sum_{l=1}^{n_{1}} L_{\mathrm{s} 1, k l} q_{11, l}(\omega),
$$




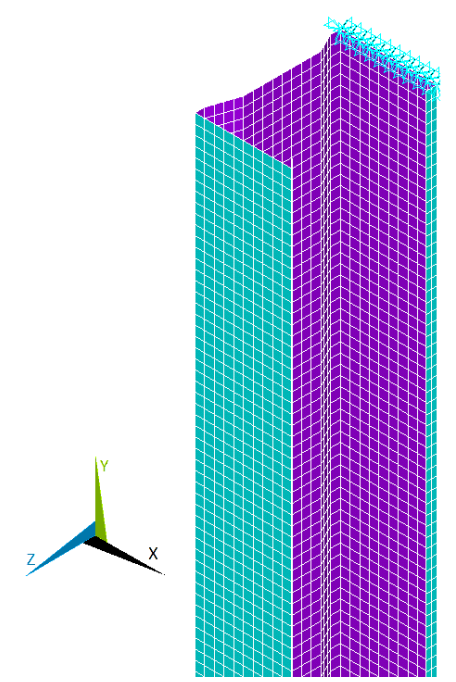

Figure 2: Finite element mesh of a single decoupled metal C-stud for a cavity depth of $75 \mathrm{~mm}$. Structural shell elements are used. As indicated on the top right part of the figure, the boundary displacements (not the rotations) are restrained at one of the flange edges. At the bottom of the stud (not shown in this figure), the boundary displacements (not the rotations) are restrained at the opposite flange.

where

$$
L_{\mathrm{s} 1, k l}:=\rho_{\mathrm{a}} \omega^{2} L_{\mathrm{f} 1, l k}
$$

Note that $L_{\mathrm{s} 1, k l}$ equals element $(k, l)$ of the coupling matrix $\mathbf{L}_{\mathrm{s} 1}$ from the overall system of equations (5), and that $L_{\mathrm{f} 1, l k}$ follows from (24-26).

Closed form expressions for the elements of the fluid-structure coupling matrices involving the second wall leaf and the cavity, i.e., $\mathbf{L}_{\mathrm{f} 2}$ and $\mathbf{L}_{\mathrm{s} 2}$ from (5), can be obtained following the same lines as for the first wall leaf and the cavity.

\subsubsection{Finite element model of the studs}

In double-leaf walls where the leafs are attached to a common stud frame, the dynamic behavior of the studs has a large influence on the overall sound insulation. Two types of stud are very commonly employed in the construction industry: wooden studs with a solid rectangular cross-section, and metal studs with a thin-walled open cross section, such as metal C-studs. The dynamic mechanical behavior of both types of stud is fundamentally different. The cross section of rectangular studs is relatively rigid, and therefore they can be taken to satisfy Euler-Bernouilli or Timoshenko beam theory. Whether this is also the case for $\mathrm{C}$-studs depends on the rigidity of the cross section. Legault and Atalla [50, 51] showed that the influence of stiff aluminum C-studs with a wall thickness of $3.2 \mathrm{~mm}$ and a web height of $50.8 \mathrm{~mm}$ on the sound insulation of a particular double-leaf wall can be well accounted for up to about $2000 \mathrm{~Hz}$ by taking the studs to be beam stiffeners. However, the studs that are employed in the construction industry are much more flexible; it typically concerns metal C-studs with a wall thickness of $0.6 \mathrm{~mm}$ and a web height of up to $100 \mathrm{~mm}$ and more. It is this type of stud that is considered here, and it has been confirmed in the present study that such studs show, next to a beam-like behavior, also substantial cross-sectional deformation, even at low frequencies.

Therefore, in the present work, the studs are modeled numerically, using the finite element method [52]. As the considered studs are thin-walled profiles, they can be adequately modelled with structural shell elements. The particular element type that is employed here is the Shell181 element from the Ansys finite element software; it is a four-node element with six degrees of freedom - three displacements and three rotations - at each node. As the wavelength of deformation at high frequencies of flexible studs is small, a fine mesh is needed; in the present work, an element edge length of $5 \mathrm{~mm}$ is employed (Fig. 2). In the construction industry, metal studs of a double-leaf wall are usually placed in flexible rails that are mounted on the floor and the ceiling. These rails are omitted from the present model. Instead, 'hinged' boundary conditions are assumed at the ends of each stud; they are implemented by 
restraining all nodal displacements (but not the rotations) at one flange at the bottom and at the opposite flange at the top (Fig. 2).

This finite element model represents a single stud that is decoupled from the leafs (and obviously also the cavity, since any interaction between the cavity and the leafs is neglected). The model is then employed for computing the natural frequencies and mass-normalized displacement mode shapes of the decoupled stud. These numerically computed mode shapes are taken to be the basis functions which appear in Eq. (4). The corresponding generalized coordinates are then the modal amplitudes of the stud. The equation of motion of a particular stud $l$ therefore reads

$$
\mathbf{D}_{\mathrm{st}, l} \mathbf{q}_{\mathrm{st}, l}=\tilde{\mathbf{f}}_{\mathrm{st}, l}
$$

where $\mathbf{D}_{\mathrm{st}, l}$ is a diagonal matrix with entries

$$
D_{\mathrm{st}, l, k k}=-\omega^{2}+\omega_{\mathrm{st}, l, k}^{2}\left(1+i \eta_{\mathrm{st}, l, k}\right)
$$

with $\omega_{\mathrm{st}, l, k}$ the numerically computed angular natural frequency of mode $k$ of stud $l$, and $\eta_{\mathrm{st}, l, k}$ the corresponding structural damping loss factor. When the loading onto the stud is coupled within the modal load vector $\tilde{\mathbf{f}}_{\mathrm{st}, l}$, (30) is fully consistent with block row $3+l$ of the equation of motion of the entire wall system, Eq. (5). Note that, when all studs are identical, the numerical modal analysis needs to be performed only once, i.e., for a single decoupled stud.

\subsubsection{Stud-leaf interaction}

A stud is loaded only through the connections with the wall leafs. For the walls that are considered in the present work, the plates constituting the leafs are screwed directly into the studs at a few points over the height of the stud. Typically, the vertical joints between the plates (not modelled here) coincide with the center of the flange of a stud. Since both plates are screwed into the same common stud, the screws connecting stud and leaf occur in pairs of two. The screws constituting a pair have the same height and a relatively small horizontal distance between them; a distance of $1 \mathrm{~cm}$ is taken in the present paper. The stud-leaf connections are modelled by rigidly coupling the out of plane displacements at the screw locations (Fig. 3). This implies that in the model, the finite stiffness of the screws is neglected, and the studs and leafs can deform independently everywhere except at the screw locations. Since the studs themselves are a lot more flexible than the plate-stud connection by the screws, the assumption of rigid connections is expected to have only a minor influence on the overall sound insulation compared to wall setups with rigid wooden studs as in [23] where the connections between the rigid studs and the plates are modelled with springs. This connection model was found to result in a good prediction accuracy as discussed further. Alternative connection models, where the stud and the leaf are connected along the entire centerline of the stud flange, or even at the entire stud-leaf interface area, were also considered but they result in a wall system that is too stiff.

Because rigid point couplings between the leafs and a stud are considered, their mutual interaction can be modelled by means of constraint equations rather than through explicitly accounting for the interface forces. The interaction between leaf 1 and stud $l$ for example results in the following set of constraint equations:

$$
\forall s: u_{\mathrm{st}, l}\left(\mathbf{x}_{l 1 s}\right)=u_{11}\left(\mathbf{x}_{l 1 s}\right)
$$

where $\mathbf{x}_{l 1 s}$ denotes the location at which screw number $s$ connects stud number $l$ to the first wall leaf. With the decompositions (1) and (4), the constraints can be expressed in terms of the generalized coordinates:

$$
\forall s: \phi_{\mathrm{st}, l}\left(\mathbf{x}_{l 1 s}\right) \mathbf{q}_{\mathrm{st}, l}=\phi_{11}\left(\mathbf{x}_{l 1 s}\right) \mathbf{q}_{11}
$$

which can be written in matrix form as

$$
\tilde{\mathbf{C}}_{l} \mathbf{q}_{\mathrm{st}, l}+\tilde{\mathbf{C}}_{11, l} \mathbf{q}_{11}=\mathbf{0}
$$

where $\phi_{\mathrm{st}, l}\left(\mathbf{x}_{l 1 s}\right)$ equals row number $s$ of $\tilde{\mathbf{C}}_{l}$, and $-\phi_{11}\left(\mathbf{x}_{l 1 s}\right)$ equals row number $s$ of $\tilde{\mathbf{C}}_{11, l}$.

Consider for a moment the equations of motion of leaf 1 (11) and of stud $l(30)$ without any external loading

$$
\left[\begin{array}{cc}
\mathbf{D}_{11} & \mathbf{0} \\
\mathbf{0} & \mathbf{D}_{\mathrm{st}, l}
\end{array}\right]\left[\begin{array}{c}
\mathbf{q}_{11} \\
\mathbf{q}_{\mathrm{st}, l}
\end{array}\right]=\mathbf{0}
$$




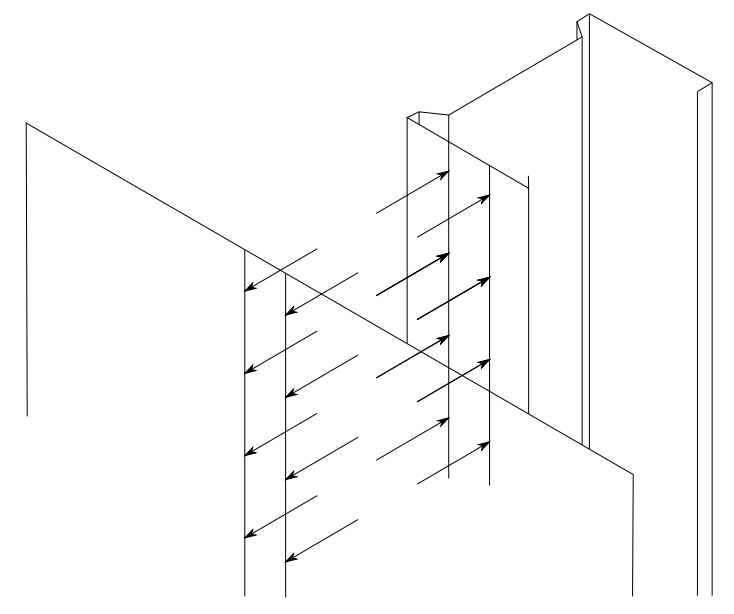

Figure 3: Coupling of the out-of-plane displacements of a metal C-stud and the leaf at four pairs of discrete screw positions. The screws constituting a pair have the same height and they have a horizontal distance of $1 \mathrm{~cm}$.

The constraints of Eq. (34) can be incorporated into the equations of motion of Eq. (35), e.g. with the technique of Lagrange multipliers [53, Sec. 13.2]. Suppose for a moment that the loading by the first wall leaf is the only loading acting on stud $l$, and vice versa. This results in

$$
\left[\begin{array}{ccc}
\mathbf{D}_{11} & \mathbf{0} & \tilde{\mathbf{C}}_{11, l}^{\mathrm{T}} \\
\mathbf{0} & \mathbf{D}_{\mathrm{st}, l} & \tilde{\mathbf{C}}_{l}^{\mathrm{T}} \\
\tilde{\mathbf{C}}_{11, l} & \tilde{\mathbf{C}}_{l} & \mathbf{0}
\end{array}\right]\left[\begin{array}{c}
\mathbf{q}_{\mathrm{l} 1} \\
\mathbf{q}_{\mathrm{st}, l} \\
\boldsymbol{\lambda}_{\mathbf{l} 1, l}
\end{array}\right]=\mathbf{0}
$$

where the Lagrange multipliers have been collected into the vector $\lambda_{11, l}$.

More generally, the system of equations (34) can be extended to include all stud-leaf interactions:

$$
\mathbf{C}_{11} \mathbf{q}_{11}+\mathbf{C}_{11} \mathbf{q}_{12}+\sum_{l=1}^{\mathrm{n}_{\mathrm{st}}} \mathbf{C}_{l} \mathbf{q}_{\mathrm{st}, l}=\mathbf{0} .
$$

Obviously, in any row of this system of equations, only two of the $\mathbf{C}$ matrices have nonzero entries. Application of the Lagrange multiplier technique to the entire wall system then leads to the overall system of equations, (5).

\subsubsection{Reduction of the dynamic stiffness matrix}

Eq. (5) represents a large system of equations which describes the vibro-acoustic behavior of the wall in terms of all generalized degrees of freedom involved. Since the external loading by the sound pressure in the adjoining rooms is only applied at the leafs, one can eliminate all interior degrees of freedom from (5) prior to specifying the external loading. The resulting reduced system of equations is denoted as

$$
\mathbf{D}_{\mathrm{d}}^{\prime} \mathbf{q}^{\prime}=\mathbf{f}^{\prime}
$$

where

$$
\mathbf{D}_{\mathrm{d}}^{\prime}:=\left[\begin{array}{cc}
\mathbf{D}_{11} & \mathbf{0} \\
\mathbf{0} & \mathbf{D}_{12}
\end{array}\right]+\mathbf{D}_{\mathrm{fsi}}^{\prime}+\mathbf{D}_{\mathrm{st}}^{\prime} \quad \text { and } \quad \mathbf{q}^{\prime}:=\left[\begin{array}{l}
\mathbf{q}_{11} \\
\mathbf{q}_{12}
\end{array}\right] .
$$

In this equation, $\mathbf{D}_{\mathrm{fsi}}^{\prime}$ is a fluid-structure interaction matrix resulting from the elimination of the cavity pressure degrees of freedom from (5),

$$
\mathbf{D}_{\mathrm{fsi}}^{\prime}=\omega^{2}\left[\begin{array}{l}
\mathbf{L}_{\mathrm{f}, 1} \\
\mathbf{L}_{\mathrm{f}, 2}
\end{array}\right] \mathbf{D}_{\mathrm{cav}}^{-1}\left[\mathbf{L}_{\mathrm{s}, 1} \quad \mathbf{L}_{\mathrm{s}, 2}\right]
$$


and $\mathbf{D}_{\mathrm{st}}^{\prime}$ is a matrix resulting from the elimination of the stud displacement degrees of freedom and the Lagrange multipliers from (5),

$$
\mathbf{D}_{\mathrm{st}, \mathrm{cp}}^{\prime}=\left[\begin{array}{l}
\mathbf{C}_{11}^{T} \\
\mathbf{C}_{12}^{T}
\end{array}\right]\left(\sum_{l=1}^{\mathrm{n}_{\mathrm{st}}} \mathbf{C}_{l} \mathbf{D}_{\mathrm{st}, l}^{-1} \mathbf{C}_{l}^{T}\right)\left[\begin{array}{ll}
\mathbf{C}_{11} & \mathbf{C}_{12}
\end{array}\right] .
$$

\subsection{Transmission suite model}

In the previous section, the deterministic model of the double-leaf wall was described in full detail. In the present section, this wall model is coupled to models for the sound fields in the adjoining rooms.

These sound fields are modelled as diffuse, i.e., they are taken to be random pressure fields which are composed of a large number of statistically independent plane waves, the spatial phase of which is uniformly distributed and independent from the amplitude [54]. Although a diffuse sound field represents a random ensemble of rooms, it can also be used for modeling the sound field in one specific room at high frequencies, i.e., at frequencies where the modal density of the room is typically larger than unity. This is because at high frequencies, the variance of the total sound energy across the random ensemble representing the diffuse field is low, and therefore the mean total energy of the ensemble will be close to the total energy in any particular member of the random ensemble [55]. At low frequencies however, this is not the case. When a diffuse field model is employed at low frequencies as in the present paper, it therefore represents an ensemble of sound fields rather than one particular sound field, and considerable variation can occur when comparing the mean total sound energy of the diffuse model with the total sound energy in one particular sound field.

A perfectly diffuse sound field requires diffusely reflecting boundaries, i.e., boundaries that reflect an incident plane wave in a random direction that is statistically independent of the direction of incidence. When boundary reflections are specular rather than diffuse, the mean energy density will not be uniform anymore because of interference between incoming and reflected sound waves, but this effect can be accounted for [56]. Further generalization is possible by establishing a harmonic linear relationship between the mean total energy in the diffuse field and the mean squared amplitude of the blocked reverberant forces at the locations where the loading that generates the diffuse field, occurs. This relationship is termed the diffuse field reciprocity relationship [6, 45]. It forms the basis of a hybrid method of analysis, in which a built-up system consisting of components that are modelled as deterministic and components that are modelled as diffuse can be rigorously analyzed. This method is termed the hybrid DETerministic-Statistical Energy Analysis (DET-SEA) method [4, 57, 58].

Within the hybrid DET-SEA framework, a transmission suite (room-wall-room) model has been developed by Reynders et al. $[5,59,60]$. In this model, which is adopted also in the present work, the rooms are taken to carry a diffuse field, while the wall is modeled deterministically. The features of the model that are essential for the present analysis are detailed in the remainder of this section.

The hybrid model contains two diffuse (SEA) subsystems - the sending and receiving rooms - and, in the context of a sound transmission analysis, the quantity of interest is the so-called coupling loss factor between both rooms. If in a stationary situation, the sound power flow from room 1 to room 2 (so through the wall) is denoted as $P_{12}$, the total acoustic energy of room $k$ is denoted as $E_{k}$ and its modal density (i.e., the expected number of modes per unit radial bandwidth) as $n_{k}$, then the coupling loss factor $\eta_{12}$ is defined as [55]

$$
\eta_{12}:=\frac{\omega n_{1}}{P_{12}}\left(\frac{E_{1}}{n_{1}}-\frac{E_{2}}{n_{2}}\right) .
$$

Although the coupling loss factor is a random quantity (because the sound fields in the rooms are random, diffuse fields), only its mean value will be of interest in the present analysis as the intention is to predict the mean sound transmission loss of the wall. The coupling loss factor relates directly to the sound transmission coefficient, $\tau$, which is defined as the ratio between the power flow $P_{12}$ from room 1 to room 2 through the wall, and the incident sound power on the wall in room 1 . The relationship reads [61]

$$
\tau=\frac{4 V_{1} \omega}{L_{\mathrm{x}} L_{\mathrm{y}} c} \eta_{12},
$$


where $V_{1}$ denotes the volume of the sending room. The sound transmission loss (or airborne sound insulation) of the wall then immediately follows from

$$
R:=10 \log \frac{1}{\tau}=10 \log \frac{L_{\mathrm{x}} L_{\mathrm{y}} c}{4 V_{1} \omega \eta_{12}} .
$$

Within the hybrid DET-SEA framework, the coupling loss factor $\eta_{12}$ can be rigorously evaluated by considering the interaction between the wall and the direct field response of the rooms at their interfaces $[4,5,62]$. The direct field response of a room is the sound field that would occur if the room would be of infinite extent, in other words, if the room would behave as an acoustic half-space as seen from the room-wall interface when that interface is embedded in an infinite planar baffle. The related acoustic dynamic stiffness matrix is then termed the direct field dynamic stiffness matrix of the room. For room 1 for example, this matrix describes the relationship between the displacements and forces at the interface with the first wall leaf:

$$
\mathbf{D}_{\operatorname{dir} 1} \mathbf{q}_{11}=\tilde{\mathbf{f}}_{\operatorname{dir} 1}
$$

The components of $\tilde{\mathbf{f}}_{\text {dir } 1}$ denote the forces acting on the (generalized) degrees of freedom of the first wall leaf $\mathbf{q}_{11}$ due to the pressure field in the acoustic half-space. The direct field dynamic stiffness matrix $\mathbf{D}_{\text {dir } 1}$ can be numerically computed via evaluation of the Rayleigh integral, e.g. using a wavelet discretization of the baffled interface; details of this approach are provided by Langley [63].

Once the direct field acoustic dynamic stiffness matrices of both rooms have been computed, the coupling loss factor is obtained from [4]

$$
\eta_{12}=\frac{2}{\pi \omega n_{1}} \sum_{r s} \operatorname{Im}\left(\mathbf{D}_{\operatorname{dir} 2, r s}^{\prime}\right)\left(\mathbf{D}_{\mathrm{tot}}^{-1} \operatorname{Im}\left(\mathbf{D}_{\mathrm{dir} 1}^{\prime}\right) \mathbf{D}_{\mathrm{tot}}^{-\mathrm{H}}\right)_{r s}
$$

where

$$
\mathbf{D}_{\text {tot }}:=\mathbf{D}_{\mathrm{d}}^{\prime}+\mathbf{D}_{\text {dir } 1}^{\prime}+\mathbf{D}_{\text {dir } 2}^{\prime}
$$

$\mathbf{D}_{\mathrm{d}}^{\prime}$ denotes the reduced dynamic stiffness matrix of the wall as defined in (39), and $\mathbf{D}_{\mathrm{dir} 1}^{\prime}$ and $\mathbf{D}_{\mathrm{dir} 2}^{\prime}$ are the direct field acoustic dynamic stiffness matrices expressed in terms of the reduced wall coordinate vector $\mathbf{q}^{\prime}$.

The computational efficiency can be further increased by exploiting the block structure of the direct field acoustic dynamic stiffness matrices:

$$
\mathbf{D}_{\operatorname{dir} 1}^{\prime}=\left[\begin{array}{cc}
\mathbf{D}_{\operatorname{dir} 1} & \mathbf{0} \\
\mathbf{0} & \mathbf{0}
\end{array}\right] \quad \text { and } \quad \mathbf{D}_{\operatorname{dir} 2}^{\prime}=\left[\begin{array}{cc}
\mathbf{0} & \mathbf{0} \\
\mathbf{0} & \mathbf{D}_{\mathrm{dir} 2}
\end{array}\right]
$$

Equation (46) can be elaborated as

$$
\eta_{12}=\frac{2}{\pi \omega n_{1}} \sum_{r s} \operatorname{Im}\left(\mathbf{D}_{\mathrm{dir} 2, r s}\right)\left(\left(\mathbf{D}_{\mathrm{tot}}^{-1}\right)_{2,1} \operatorname{Im}\left(\mathbf{D}_{\mathrm{dir} 1}\right)\left(\mathbf{D}_{\mathrm{tot}}^{-1}\right)_{2,1}^{\mathrm{H}}\right)_{r s}
$$

where $\left(\mathbf{D}_{\text {tot }}^{-1}\right)_{2,1}$ denotes the left bottom block matrix of $\mathbf{D}_{\text {tot }}^{-1}$ which can be computed using the following inversion property of $2 \times 2$ block-structured matrices.

$$
\left(\mathbf{A}^{-1}\right)_{2,1}=-\left(\mathbf{A}_{2,2}-\mathbf{A}_{2,1} \mathbf{A}_{1,1}^{-1} \mathbf{A}_{1,2}\right)^{-1} \mathbf{A}_{2,1} \mathbf{A}_{1,1}^{-1}
$$

where $\mathbf{A}_{i, j}$ represents the block with index (i,j) of the matrix $\mathbf{A}$.

\section{Experimental validation}

The model that was presented in the previous section is employed in the present section for predicting the airborne sound insulation of 14 different double-leaf plasterboard walls that each have a common flexible metal stud frame. The walls differ by the cavity depth $(40,45,50$ or $100 \mathrm{~mm})$, the material inside the cavity (air or mineral wool) and the number of plasterboard layers in the leafs (single, double or triple plating). In order to assess the accuracy of the sound insulation predictions, they are compared with the results from an extensive measurement campaign [2]. 


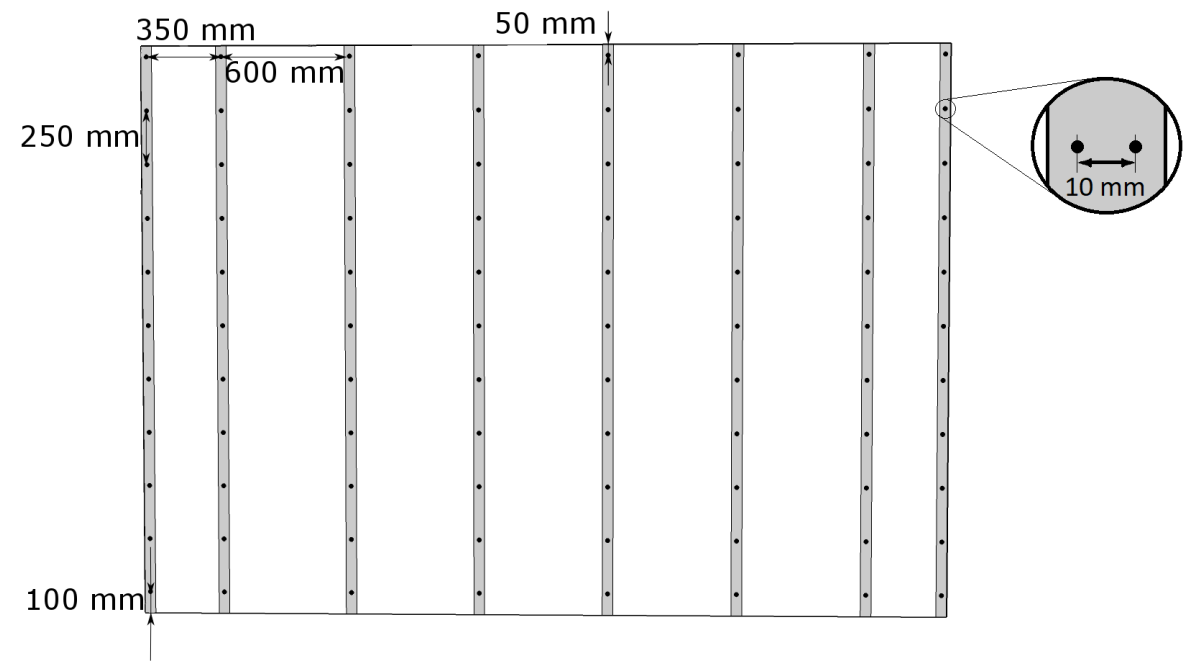

Figure 4: Positioning of studs and screw pairs within a wall. Eleven screw pairs are situated on each side of the stud with a vertical distance of 250 $\mathrm{mm}$ starting $100 \mathrm{~mm}$ from the bottom. Center to center spacing of the studs is $600 \mathrm{~mm}$ for the middle studs and $350 \mathrm{~mm}$ at the sides.

\subsection{Wall and room properties}

The test opening of the transmission suite that is considered in this validation study has a width of $3.75 \mathrm{~m}$ and a height of $2.65 \mathrm{~m}$. The transmission rooms have a volume of $100 \mathrm{~m}^{3}$ each. The frame consists of steel C-studs with a flange width of $50 \mathrm{~mm}$ and a wall thickness of $0.6 \mathrm{~mm}$. The web height of the studs equals the cavity depth. As discussed previously, the horizontal rails in which the studs are placed are omitted from the model and instead 'hinged' boundary conditions are assumed at the stud ends. All walls contain eight studs in total, two of which are placed against the vertical edges of the test opening, and six in between. The stud and screw positions are depicted in Fig. 4. The center to center distance between two adjoining studs equals $350 \mathrm{~mm}$ at the vertical edges of the test opening and $600 \mathrm{~mm}$ in between. In order to connect a plasterboard plate to a metal stud, eleven screws are used along the vertical plate edge. Screws start at $100 \mathrm{~mm}$ from the bottom edge and end at $50 \mathrm{~mm}$ from the top edge of a plate. The vertical distance between two screws is $250 \mathrm{~mm}$. The horizontal distance between two screws in a pair is $10 \mathrm{~mm}$ in the model.

The plasterboard plates have a nominal density of $720 \mathrm{~kg} / \mathrm{m}^{3}$ [2]. The Young's modulus and the Poisson's ratio of the plasterboard plates are chosen to match the experimental coincidence frequencies in two of the sound transmission tests, since no experimental values are available. This results in a Young's modulus of 2.5 GPa and a Poisson's ratio of 0.3 [64]. A constant damping loss factor of 0.03 is assumed for the plasterboard, as in [64]. The assumed material properties for the galvanized steel studs are: a Young's modulus of $210 \mathrm{GPa}$, a density of $7800 \mathrm{~kg} / \mathrm{m}^{3}$, a Poisson's ratio of 0.25 and a damping loss factor of 0.02 . For air the following material properties are assumed: a density of $1.21 \mathrm{~kg} / \mathrm{m}^{3}$ and a sound speed of $340 \mathrm{~m} / \mathrm{s}$. The flow resistivity of the mineral wool, used as sound absorbent in the cavity, was measured to be $5000 \mathrm{~N} / \mathrm{sm}^{4}$ [2].

\subsection{Sound insulation predictions}

For each of the 14 considered walls, the airborne sound transmission loss is computed at 320 frequency lines spanning the 1/3-octave bands with center frequencies between 50 and $4000 \mathrm{~Hz}$. These frequency lines therefore coincide with the 1/48-octave band center frequencies. The harmonic sound transmission coefficients, $\tau$, are subsequently averaged over each $1 / 3$ octave band. The total computation time depends on the number of generalized degrees of freedom in Eq. (5) that are necessary to accurately describe the dynamic behavior of the considered wall. The calculations have been performed on a single personal computer with a $2.7 \mathrm{GHz}$ Intel Core i7 processor and 16 GB RAM. The time needed to compute the transmission loss at all 320 frequency lines ranged from 45 minutes to 1 hour, depending on the specific wall configuration. 

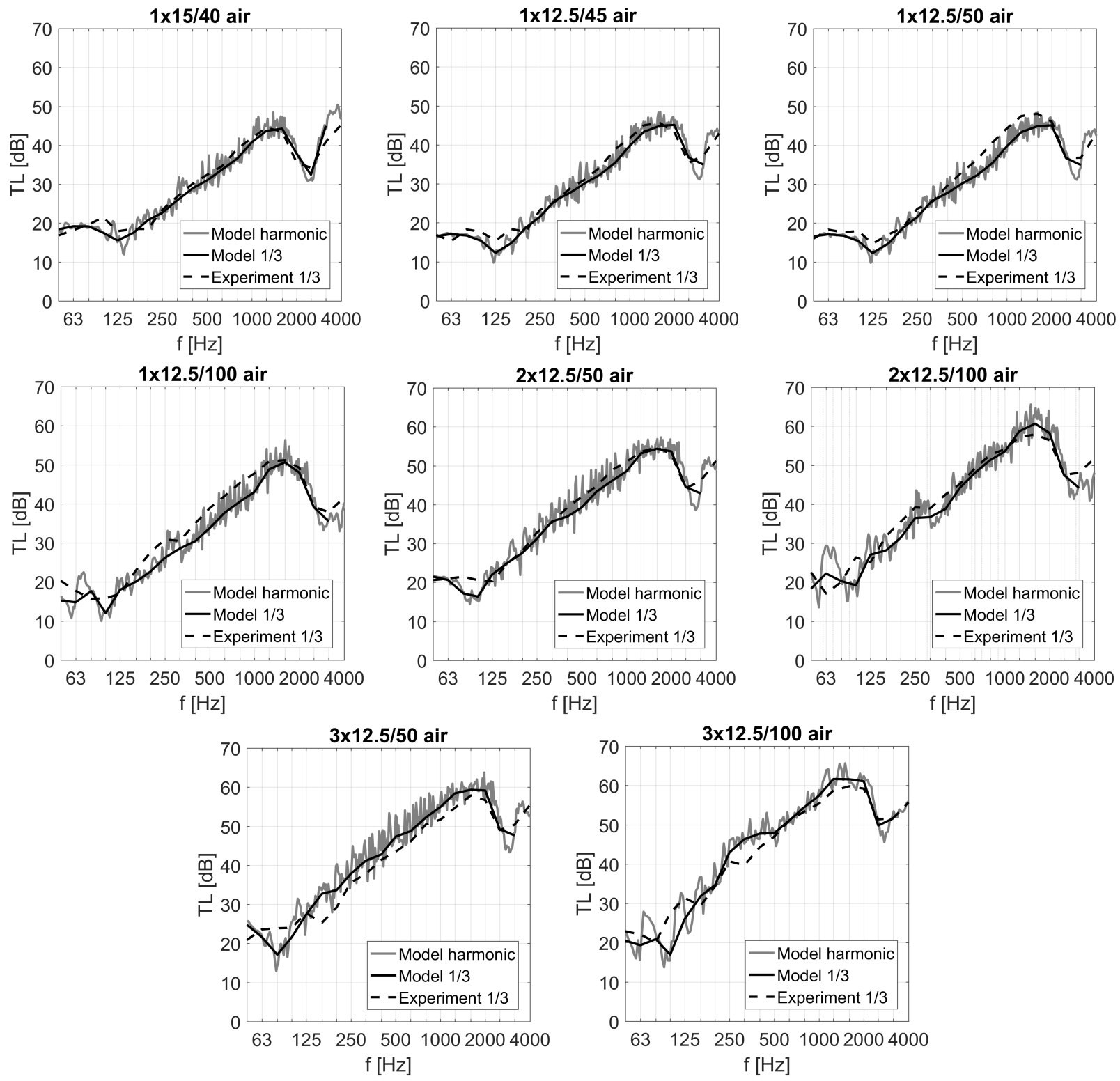

Figure 5: Predicted versus measured airborne sound insulation of a set of studded double-leaf plasterboard walls. Cavity filled with air. 

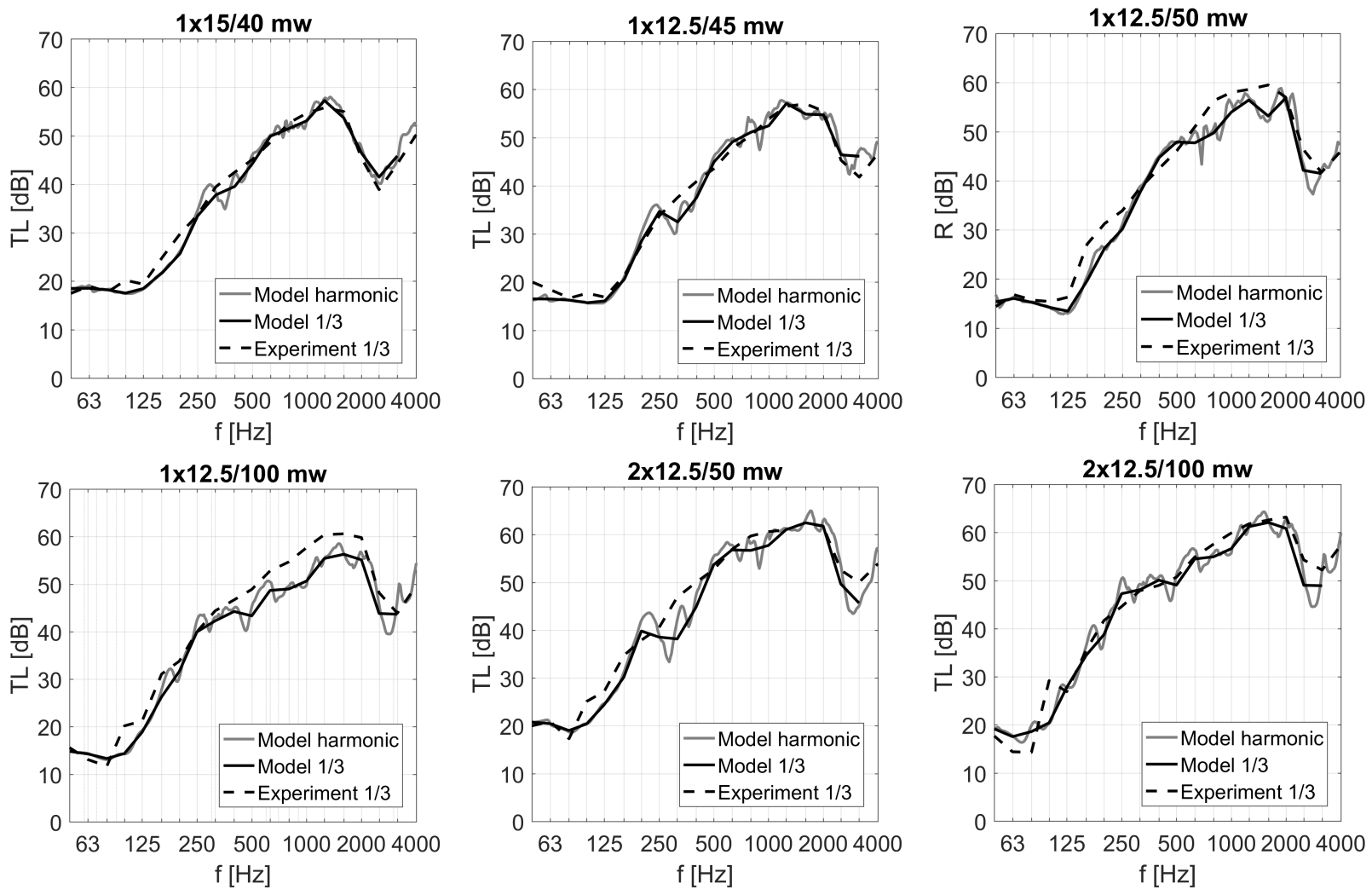

Figure 6: Predicted versus measured airborne sound insulation of a set of studded double-leaf plasterboard walls. Cavity filled with mineral wool. 
The 14 walls that are considered in this study are labeled by the combination of the number of platings constituting one leaf, the thickness of a single plating in $\mathrm{mm}$, the cavity depth in $\mathrm{mm}$ and the type of cavity filling (air or mineral wool). For example, the wall labeled as $2 \times 12.5 / 50 \mathrm{mw}$ has leafs with double plasterboard plating, an individual plating thickness of $12.5 \mathrm{~mm}$ and, a cavity with a depth of $50 \mathrm{~mm}$ and mineral wool as filling. In Table 1, the sound insulation predictions are summarized in terms of the single-number ratings according to the ISO 717 - $1: 1996$ standard [65]. The transmission loss curves as a function of frequency are displayed in Figs. 5 and 6.

\begin{tabular}{l|c|c}
\hline \hline Wall type & $\mathrm{R}_{\mathrm{W}}\left(\mathrm{C} ; \mathrm{C}_{\text {tr }}\right)_{\exp } \mathrm{dB}$ & $\mathrm{R}_{\mathrm{W}}\left(\mathrm{C} ; \mathrm{C}_{\text {tr }}\right)_{\text {model }} \mathrm{dB}$ \\
\hline $1 \times 15 / 40$ air & $34(-1 ;-5)$ & $33(-1 ;-5)$ \\
$1 \times 12.5 / 45$ air & $34(-2 ;-6)$ & $33(-2 ;-6)$ \\
$1 \times 12.5 / 50$ air & $34(-2 ;-6)$ & $33(-2 ;-6)$ \\
$1 \times 12.5 / 100$ air & $38(-1 ;-6)$ & $36(-2 ;-7)$ \\
$2 \times 12.5 / 50$ air & $42(-2 ;-7)$ & $41(-2 ;-8)$ \\
$2 \times 12.5 / 100$ air & $47(-2 ;-6)$ & $44(-1 ;-7)$ \\
$3 \times 12.5 / 50$ air & $45(-2 ;-7)$ & $47(-2 ;-8)$ \\
$3 \times 12.5 / 100$ air & $49(-2 ;-7)$ & $49(-2 ;-7)$ \\
$1 \times 15 / 40 \mathrm{mw}$ & $42(-2 ;-7)$ & $40(-3 ;-8)$ \\
$1 \times 12.5 / 45 \mathrm{mw}$ & $41(-3 ;-9)$ & $41(-4 ;-10)$ \\
$1 \times 12.5 / 50 \mathrm{mw}$ & $42(-3 ;-10)$ & $40(-3 ;-9)$ \\
$1 \times 12.5 / 100 \mathrm{mw}$ & $46(-3 ;-9)$ & $43(-1 ;-11)$ \\
$2 \times 12.5 / 50 \mathrm{mw}$ & $50(-2 ;-8)$ & $46(-2 ;-8)$ \\
$2 \times 12.5 / 100 \mathrm{mw}$ & $52(-3 ;-8)$ & $50(-4 ;-11)$ \\
\hline \hline
\end{tabular}

Table 1: Wall type with corresponding experimental results and predictions of the single number ratings.

\subsection{Physical interpretation and comparison with experiments}

In Table 1 and Figs. 5 and 6, the prediction results are also compared with data from an extensive experimental study at the TNO TPD Delft Acoustics Laboratory as reported by Vermeir and Gerretsen [2]. The tests were conducted according to the ISO 140 - $3: 1995$ standard [66], measuring the transmission losses in both directions and averaging the sound pressure levels over 8 microphone positions in each room.

In general, a very good agreement between the model predictions and the experimental results can be observed. This is also the case at low frequencies, although the sound fields in the rooms are modelled as diffuse while in a single experiment, the modal behavior of the rooms will be important. The ISO 12999 - $1: 2014$ standard [67] defines an average experimental reproducibility for the single number ratings $R_{\mathrm{w}}, \mathrm{R}_{\mathrm{w}}+\mathrm{C}$ and $\mathrm{R}_{\mathrm{w}}+\mathrm{C}_{\mathrm{tr}}$ as respectively 2.0, 2.1 and $2.4 \mathrm{~dB}$ for a $95 \%$ confidence interval. From Table 1 it can be concluded that for nearly all walls, the predicted single-number ratings differ from the experimental values by 0 to $2 \mathrm{~dB}$ which is close to the average reproducibility with a coverage probability of $95 \%$ for experiments. Furthermore, it can be observed from Figs. 5 and 6 that in most setups and at most frequencies, the sound insulation deviates at most $2 \mathrm{~dB}$ from the experimental results. This constitutes a good result taking into account that the Just Noticeable Differences (JNDs) for human hearing range between 0.5 and $1 \mathrm{~dB}$ in the frequency range of interest [68].

An important resonance phenomenon for double-leaf walls is the so-called mass-spring-mass resonance. For an infinite wall without studs, the leafs then vibrate as rigid masses and compress the cavity fluid. For a finite studded wall, the mass-spring-mass resonance cannot fully develop due to restrained motion at the leaf edges and the presence of the studs. Nevertheless, a dip in the airborne sound insulation is usually observed around the theoretical massspring-mass resonance frequency, especially when there is no absorbent present in the cavity. This is also the case for the walls considered here. As can be observed in Figs. 5 and 6, the mass-spring-mass resonance dip is situated between $71 \mathrm{~Hz}$ (for wall 3x12.5/100 air) and $182 \mathrm{~Hz}$ (for wall 1x12.5/45 air). From these figures, it can also be observed that the predicted sound insulation around the mass-spring-mass resonance frequency agrees very will with the experimental findings.

Because of the good prediction performance, the model can also be employed for investigating the influence of the studs on the sound insulation of the entire wall. This is achieved by making a prediction in which the studs are 
present, and another prediction in which the studs are not present. The result of such a prediction is displayed in Fig. 7 for wall 1x12.5/50 mw. Above the mass-spring-mass resonance frequency, the presence of the studs results in a reduction of the airborne sound insulation. However, at and below the mass-spring-mass resonance frequency, the presence of the studs is beneficial: they stiffen the wall system and attenuate the airborne resonance phenomenon.

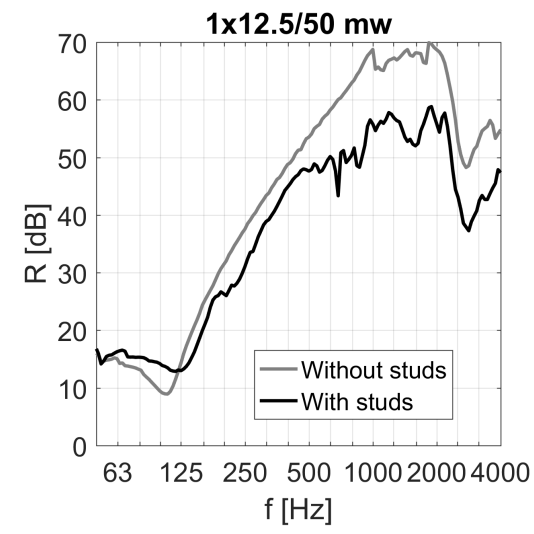

Figure 7: Comparison of the airborne sound insulation for wall 1x12.5/50 mw with and without studs. The theoretical mass-spring-mass resonance frequency is $115 \mathrm{~Hz}$.

The prediction model can also be employed for investigating the relative contributions of the airborne transmission through the cavity and the structural transmission through the studs. This is achieved by removing the studs or the cavity from the prediction model, respectively. The result of such a prediction is displayed in Fig. 8 for wall 1x12.5/50 mw. While the airborne transmission dominates at low frequencies, the structural transmission starts to dominate from about $400 \mathrm{~Hz}$ on. This is the so-called bridge frequency. Above the bridge frequency, the sound insulation increases less strongly with frequency than would be expected for a wall without frame, see also Fig. 7.

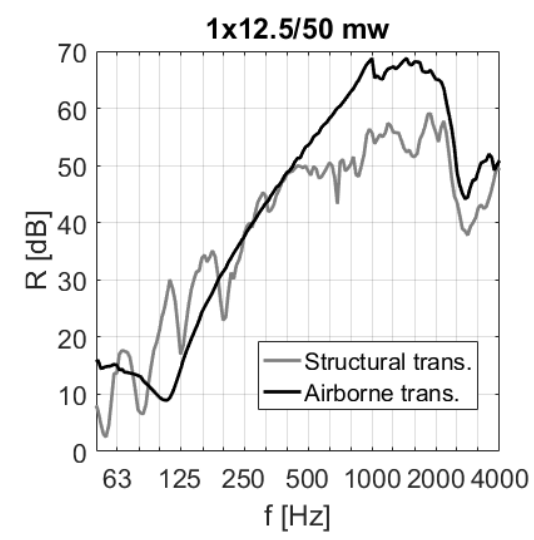

Figure 8: Sound insulation prediction for wall $1 \times 12.5 / 50 \mathrm{mw}$ with airborne transmission through the cavity only, and structural transmission through the studs only.

The structural transmission through the studs only and the transmission through the cavity only is often approximately computed separately and then combined into a global transmission loss by adding the separate transmission coefficients, $\tau$, as in $[19,20]$. The result of such an approximate approach is shown in Fig. 9 for wall $1 \times 12.5 / 50$ $\mathrm{mw}$ and it is compared to the results of the detailed model. Above the mass-spring-mass resonance frequency, the difference between both approaches is negligible. However, around the mass-spring-mass resonance, both models diverge and the sound insulation is underestimated for the model where the structural transmission and the transmission through the cavity are computed separately. This is because the interaction between the displacement field of the leafs 
coupled to the studs, and the displacement field of the leafs connected through the cavity is neglected in the model where both paths are computed separately.

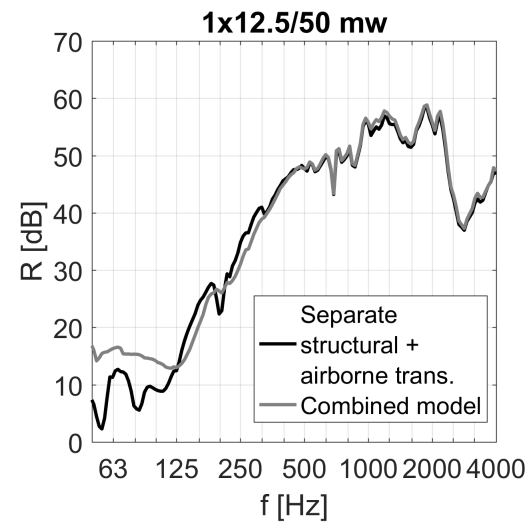

Figure 9: Sound insulation prediction for wall $1 \times 12.5 / 50 \mathrm{mw}$ with the full model, and the sum of airborne transmission through the cavity only, and structural transmission through the studs only.

At high frequencies, a pronounced dip can be observed in all transmission loss curves. This is the so-called coincidence dip. It occurs at the critical frequency of a single plasterboard plating, i.e., the lowest frequency at which the free bending wavelength on an infinite leaf matches the projected free wavelength in air. The theoretical value of the critical frequency is $2274 \mathrm{~Hz}$ for a plasterboard plate with thickness of $15 \mathrm{~mm}$, and $2728 \mathrm{~Hz}$ for a plate of $12.5 \mathrm{~mm}$ thickness. The predicted and observed coincidence dips match well. For walls $1 \times 15 / 40$ air and 1x12.5/45 air, this comes as no surprise, since the Young's modulus and Poisson ratio of the plasterboard material were determined from the coincidence dips that are observed for these two walls. More important is that in the experimental results, the critical frequency remains unchanged when the number of platings is increased from one to two or three: this justifies the assumption of perfect slip conditions at the plate-plate interfaces.

Finally, some small but systematic differences between predictions and experimental results can be observed from Figs. 5 and 6. The most pronounced ones occur for the walls with single plating and a relatively large cavity. For these walls, the sound insulation is underestimated in between the bridge frequency and the critical frequency (see, e.g., wall $1 \times 12.5 / 100$ air and wall $1 \times 12.5 / 100 \mathrm{mw}$ ). In this frequency region, structural transmission through the studs is dominant, and the predictions are very sensitive to the stud-plate coupling. The stud-plate coupling model as displayed in Fig. 3 is not entirely correct when the leafs consist of single plating. This is because the width of an individual plasterboard plate is $120 \mathrm{~cm}$ while the distance between the studs is $60 \mathrm{~cm}$, and the screw connections appear in pairs only when two individual plates connect to the same stud. In order to investigate this, an additional prediction was performed with alternating single and double point displacement couplings, as shown in Fig. 10. In this way, however, the true sound insulation is overestimated: it falls in between the results predicted by both coupling models. It can be concluded that also a single screwed connection provides some degree of rotation coupling between leaf and studs, next to the point displacement coupling.

For the walls with double plating and mineral wool in the cavity, the airborne sound insulation at the coincidence dip is underestimated. This could be due to uncertainties in the damping loss factor of the plates. By increasing the damping loss factor of the plates around the coincidence frequency to 0.045 , a better match between the observed and predicted sound insulation was found.

\subsection{Computational efficiency}

In order to assess the computational performance of the proposed model against that of a finite element model of the entire transmission suite, an additional transmission loss prediction was performed for the double-leaf plasterboard wall that was considered in [44]. The harmonic transmission loss values were computed at 3 frequency lines in each of the 1/3-octave bands from 100 to $3150 \mathrm{~Hz}$. The corresponding computation time for a detailed finite element model with frequency-dependent mesh was reported to be $29.5 \mathrm{~h}$ [44]. With the hybrid DET-SEA model presented here, it 


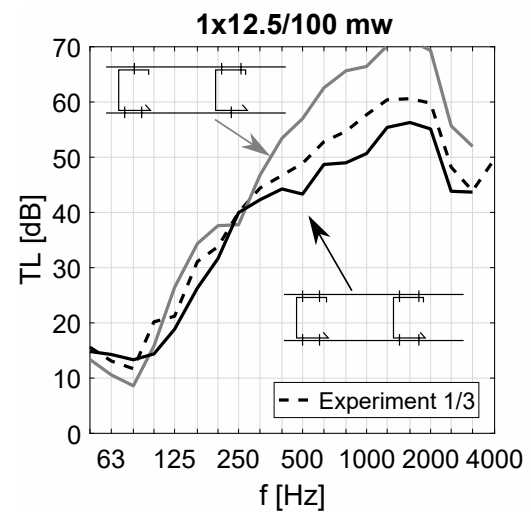

Figure 10: Sound insulation predictions for wall 1x12.5/100 mw for two different coupling models: one where the point displacement couplings always occur in pairs, and one where a single point displacement coupling alternates with a pair. The experimental results are also displayed.

took $90 \mathrm{~s}$ to compute all 48 harmonic transmission loss values with the same accuracy, which boils down to a speedup with a factor larger than 1000 .

\section{Conclusions}

In this paper, an accurate and computationally efficient model is presented for the prediction of the airborne sound insulation of studded double walls. In this model, the parts of the wall that display a low geometrical complexity, i.e. the leafs and the cavity, are modelled analytically to reduce computational cost. The studs are modelled in detail with shell finite elements, since flexible studs display a large cross-sectional deformation that cannot be captured with an analytical model. The sound fields in the rooms are modeled as diffuse, and a rigorous coupling with the deterministic wall model is achieved within the hybrid DET-SEA framework. The computation cost of this model was verified to be more than three orders of magnitude lower than that of a recently proposed detailed finite element model of the transmission suite with frequency-dependent mesh, while the prediction accuracy remains the same.

The model was extensively validated against measured transmission loss data of 14 different plasterboard walls with metal C-studs. An absolute accuracy between 0 and $2 \mathrm{~dB}$ on the single number ratings was achieved for nearly all wall types. This is within the average reproducibility of the experiments. Thanks to its prediction accuracy and computational efficiency, the model can be employed as a high-fidelity tool for the design of double-leaf wall systems.

Acknowledgments The research presented in this paper has been performed within the framework of the research project G0A7717N AcOpt: Numerical optimization of the airborne sound insulation of single and double walls, funded by the Research Foundation - Flanders (FWO), Belgium. The financial support by FWO is gratefully acknowledged.

\section{References}

[1] V. Hongisto, M. Lindgren, and R. Helenius. Sound insulation of double walls - an experimental parametric study. Acta Acustica united with Acustica, 88(6):904-923, 2002.

[2] G. Vermeir and E. Gerretsen. Onderzoek van de luchtgeluidisolatie van lichte scheidingswanden met gipskartonplaten. Technical Report PV4335/DGT-RPT-020028, K.U.Leuven and TNO, Leuven / Delft, 2002.

[3] F. Mechel and J. Royar. Hohlraumdämpfung in zweischaligen Trennwänden. WKSB, 5:23-26, 1977.

[4] P.J. Shorter and R.S. Langley. Vibro-acoustic analysis of complex systems. Journal of Sound and Vibration, 288(3):669-699, 2005.

[5] E. Reynders, R.S. Langley, A. Dijckmans, and G. Vermeir. A hybrid finite element - statistical energy analysis approach to robust sound transmission modelling. Journal of Sound and Vibration, 333(19):4621-4636, 2014.

[6] R.S. Langley. On the diffuse field reciprocity relationship and vibrational energy variance in a random subsystem at high frequencies. Journal of the Acoustical Society of America, 121(2):913-921, 2007. 
[7] L. L. Beranek and G. A. Work. Sound insulation through multiple structures containing flexible blankets. Journal of the Acoustical Society of America, 21:419-428, 1945.

[8] A. London. Transmission of reverberant sound through double walls. Journal of the Acoustical Society of America, 22(2):270-279, 1950.

[9] K. A. Mulholland, A. J. Price, and H. D. Parbrook. Transmission loss of multiple panels in a random incidence field. Journal of the Acoustical Society of America, 43:1432-1435, 1968.

[10] K. A. Mulholland, H. D. Parbrook, and A. Cummings. The transmission loss of double panels. Journal of Sound and Vibration, 6:324-334, 1967.

[11] K. A. Mulholland, A. J. Price, and H. D. Parbrook. The transmission loss of finite sized double panels in a random incidence sound field. Journal of Sound and Vibration, 8:126-133, 1968.

[12] M. Heckl. The tenth sir richard fairey memorial lecture: Sound transmission in buildings. Journal of Sound and Vibration, 77:165-189, 1981.

[13] F. Fahy. Sound and structural vibration: radiation, transmission and response. Academic Press, London, UK, 1st edition, 1985.

[14] F. Fahy. Foundations of engineering acoustics. Academic Press, San Diego, CA, 2001.

[15] A. C. Au and K. K. P. Byrne. On the insertion losses produced by plane acoustic lagging structures. Journal of the Acoustical Society of America, 82:1325-1333, 1987.

[16] A. C. Au and K. K. P. Byrne. On the insertion losses produced by acoustic lagging structures which incorporate flexurally orthotropic impervious barriers. Acustica, 70:284-291, 1990.

[17] H. Kang, J. Ih, J. Kim, and H. Kim. Prediction of sound transmission loss through multilayered panels by using Gaussian distribution of directional incident energy. Journal of the Acoustical Society of America, 107(3):1413-1420, 2000.

[18] R.J. Donato. Sound transmission through a double-leaf wall. Journal of the Acoustical Society of America, 51(3):807-815, 1972.

[19] B. Sharp. A study of techniques to increase the sound insulation of building elements. Report WR 73-5, Wyle Laboratories, June 1973.

[20] B. Sharp. Prediction methods for the sound transmission of building elements. Noise Control Engineering Journal, 11:53-63, 1978.

[21] L. Cremer and M. Heckl. Structure-borne sound: Structural vibrations and sound radiation at audio frequencies. Springer, Berlin, 2nd edition, 1988.

[22] G.F. Lin and J. M. Garrelick. Sound transmission through periodically framed parallel plates. Journal of the Acoustical Society of America, 61:1014-1018, 1977

[23] J. Brunskog. The influence of finite cavities on the sound insulation of double-plate structures. Journal of the Acoustical Society of America, 117:3727-3739, 2005.

[24] Q. Gu and J. Wang. Effect of resilient connection on sound transmission loss of metal stud double panel partitions. Chinese Journal of Acoustics, 2:113-126, 1983.

[25] J Wang, T.J. Lu, J. Woodhouse, R.S. Langley, and J. Evans. Sound transmission through lightweight double-leaf partitions: theoretical modelling. Journal of Sound and Vibration, 286:817-847, 2005.

[26] J. Poblet-Puig, A. Rodríguez-Ferran, C. Guigou-Carter, and M. Villot. The role of studs in the sound transmission of double walls. Acta Acustica united with Acustica, 95(3):555-567, 2009.

[27] J. Davy. The sound transmission of cavity walls due to studs. In Proceedings of the 1993 International Conference on Noise Control Enginnering, Leuven, Belgium, August 1993.

[28] J.L. Davy. Predicting the sound insulation of walls. Building Acoustics, 16(1):1-20, 2009.

[29] J.L. Davy. The improvement of a simple theoretical model for the prediction of the sound insulation of double leaf walls. Journal of the Acoustical Society of America, 127(2):841-849, 2010.

[30] T.E. Vigran. Sound transmission of double-leaf walls - Allowing for studs of finite stiffness in a transfer matrix scheme. Applied Acoustics, 71(7):616-621, 2010.

[31] C. Guigou-Carter, R. Foret, A. Igeleke, and S. Bailhache. Characterization of metallic studs used in gypsum board single frame walls. In Proceedings of the Acoustics 2012 Nantes Conference, Nantes, France, April 2012.

[32] P. H. White and A. Powell. Transmission of random sound and vibration through a rectangular double wall. Journal of the Acoustical Society of America, 40:821-832, 1966.

[33] R. H. Lyon and G. Maidanik. Power flow between linearly coupled oscillatorsl. Journal of the Acoustical Society of America, 34:623-639, 1962.

[34] R. H. Lyon and T. D. Scharton. Vibrational energy transmissioin a three-element structure. Journal of the Acoustical Society of America, 38:253-261, 1965

[35] A. J. Price and M. J. Crocker. Sound transmission through double panels using statistical energy analysis. Journal of the Acoustical Society of America, 47:683-693, 1970.

[36] R.J.M. Craik and R.S. Smith. Sound transmission through double leaf lightweight partitions part I: airborne sound. Applied Acoustics, 61:223-245, 2000.

[37] R. J. M. Craick and R. S. Smith. Sound transmission through double leaf lightweight partitions part II: structure-borne sound. Applied Acoustics, 61:247-269, 2000.

[38] R. Panneton and N. Atalla. Numerical prediction of sound transmission through finite multilayer systems with poroelastic materials. Journal of the Acoustical Society of America, 100(1):346-354, 1996.

[39] F. C. Sgard, N. Atalla, and J. Nicolas. A numerical model for the low-frequency diffuse field sound transmission loss of double-wall sound barriers with elastic porous lining. Journal of the Acoustical Society of America, 108:2865-2872, 2000.

[40] J. Legault and N. Atalla. Sound transmission through a double panel structure periodically coupled with vibration insulators. Journal of Sound and Vibration, 329:3082-3100, 2010.

[41] P. Davidsson, J. Brunskog, P.-A. Wernberg, G. Sandberg, and P. Hammer. Analysis of sound transmission loss of double-leaf walls in the low-frequency range using the finite element method. Building Acoustics, 11(4):239-257, 2004.

[42] J. Brunskog and P. Davidsson. Sound transmission of structures. A finite element approach with simplified room description. Acta Acustica united with Acustica, 90(5):847-857, 2004.

[43] A. Arjunan, C.J. Wang, K. Yahiaoui, D.J. Mynors, T. Morgan, and M. English. Finite element acoustic analysis of a steel stud based 
double-leaf wall. Building and Environment, 67:202-210, 2013.

[44] A. Arjunan, C.J. Wang, K. Yahiaoui, D.J. Mynors, T. Morgan, and M. English. Development of a 3D finite element acoustic model to predict the sound reduction index of stud based double-leaf walls. Journal of Sound and Vibration, 333(23):6140-6155, 2014.

[45] P.J. Shorter and R.S. Langley. On the reciprocity relationship between direct field radiation and diffuse reverberant loading. Journal of the Acoustical Society of America, 117(1):85-95, 2005.

[46] L. Meirovitch. Elements of vibration analysis. McGraw-Hill, New York, NY, 1975.

[47] H. Kuttruff. Room Acoustics. Spon Press, Oxon, UK, 5th edition, 2009

[48] T.E. Vigran. Building Acoustics. Taylor \& Francis, Oxon, UK, 2008.

[49] M.A. Delany and E.N. Bazley. Acoustical properties of fibrous materials. Applied Acoustics, 3(2):105-116, 1970.

[50] J. Legault and N. Atalla. Numerical and experimental investigation of the effect of structural links on the sound transmission of a lightweight double panel structure. Journal of Sound and Vibration, 324(3-5):712-732, 2009.

[51] J. Legault and N. Atalla. Corrigendum to "Numerical and experimental investigation of the effect of structural links on the sound transmission of a lightweight double panel structure" [J. Sound Vib. 324 (2009) 712-732]. Journal of Sound and Vibration, 332(8):2119-2121, 2013.

[52] K.J. Bathe. Finite Element Procedures. Prentice-Hall, Englewood Cliffs, NJ, second edition, 1996.

[53] R.D. Cook, D.S. Malkus, M.E. Plesha, and R.J. Witt. Concepts and applications of finite element analysis. John Wiley and Sons, fourth edition, 2002

[54] K.J. Ebeling. Statistical properties of random wave fields. In W.P. Mason and R.N. Thurston, editors, Physical acoustics Vol. XVII, pages 233-310. Academic Press, Orlando, FL, 1984

[55] R.H. Lyon and R.G. DeJong. Theory and application of statistical energy analysis. Butterworth-Heinemann, Newton, MA, second edition, 1995.

[56] R.V. Waterhouse. Interference patterns in reverberant sound fields. Journal of the Acoustical Society of America, 27(2):247-258, 1955.

[57] R.S. Langley and V. Cotoni. Response variance prediction for uncertain vibro-acoustic systems using a hybrid deterministic-statistical method. Journal of the Acoustical Society of America, 122(6):3445-3463, 2007.

[58] E. Reynders and R.S. Langley. Response probability distribution of built-up vibro-acoustic systems. Journal of the Acoustical Society of America, 131(2):1138-1149, 2012.

[59] C. Decraene, A. Dijckmans, and E.P.B. Reynders. Fast mean and variance computation of the diffuse sound transmission through finite-sized thick and layered wall and floor systems. Journal of Sound and Vibration, 422:131-145, 2018.

[60] E. Reynders, C. Van hoorickx, and A. Dijckmans. Sound transmission through finite rib-stiffened and orthotropic plates. Acta Acustica united with Acustica, 102(6):999-1010, 2016.

[61] M.J. Crocker and A.J. Price. Sound transmission using statistical energy analysis. Journal of Sound and Vibration, 9(3):469-486, 1969.

[62] R.S. Langley and J.A. Cordioli. Hybrid deterministic-statistic analysis of vibro-acoustic systems with domain couplings on statistical components. Journal of Sound and Vibration, 321(3-5):893-912, 2009.

[63] R.S. Langley. Numerical evaluation of the acoustic radiation from planar structures with general baffle conditions using wavelets. Journal of the Acoustical Society of America, 121(2):766-777, 2007.

[64] A. Dijckmans. The influence of finite dimensions on the sound insulation of double walls. In Proceedings of 7th Forum Acusticum, pages 1-6, Kraków, Poland, September 2014. CD-ROM

[65] International Organization for Standardization. ISO 717-1:1996: Acoustics - Rating of sound insulation in buildings and of building elements - Part 1: Airborne sound insulation, 1996.

[66] International Organization for Standardization. ISO 140-3:1995: Acoustics - Measurement of sound insulation in buildings and of building elements - Part 3: Laboratory measurements of airborne sound insulation of building elements, 1995.

[67] International Organization for Standardization. ISO 12999-1:2014: Acoustics - Determination and application of measurement uncertainties in building acoustics - Part 1: Sound insulation, 2014.

[68] A. W. Mills. Lateralization of high-frequency tones. Journal of the Acoustical Society of America, 32(1):132-134, 1960. 